\title{
Plant tonic, a plant-derived bioactive natural product, exhibits antifungal activity against rice blast disease
}

\begin{abstract}
The tendency towards application of natural products and botanical extracts as safer antimicrobial agents against plant pathogens has recently been increased. Plant Tonic9 (EOXSOV) is an environmentally friendly product and by its application there is no concern of resistance as it is with conventional pesticides. The goal of the present research was to determine the effect of application of this product against Magnaporthe oryzae, the causal agent of rice blast. The efficacy of plant tonic against M. oryzae was evaluated through in vitro and in vivo experiments. Under in vitro conditions, application of plant tonic at all rates $(2,3$, and $4 \mathrm{~mL} / \mathrm{L}$ ) could significantly inhibit the mycelial growth and conidial germination of fungus with the highest inhibition ( $83.63 \%$ and $95.15 \%$, respectively) recorded by the rate of $4 \mathrm{~mL}$. Plant tonic treatment ( 3 and $4 \mathrm{~mL}$ ) was more effective than fungicide treatment (propiconazole $25 \%$ EC $(0.1 \%) ; 250 \mathrm{ppm})$ to inhibit mycelial growth and conidial germination of M. oryzae. Under in vivo conditions, plant tonic application $(4 \mathrm{~mL})$ was also the most effective treatment and resulted in a significant reduction $(57.12 \%)$ of the area under the disease progress curve (AUDPC) value as compared with the control. Application of plant tonic also caused increased accumulation of phenolic compounds and higher activity of peroxidase and polyphenol oxidase enzymes than the control. The maximum amount of phenolic compounds $(0.49 \mathrm{mg}$ Gallic acid equivalent/g leaf fresh weight) and the highest activity of the enzymes (1.24 and $7.85 \mathrm{Units} / \mathrm{mL}$ for peroxidase and polyphenol oxidase, respectively) were observed in plants treated with plant tonic ( $4 \mathrm{~mL})$ and challenged with $\mathrm{M}$. oryzae as compared with other treatments. No phytotoxicity was observed in plant tonic treated rice plants when compared with the control. Results of the present study confirmed the beneficial effects of plant tonic in controlling rice blast disease. Therefore, its application may help to develop appropriate management strategies and provide with the opportunity to have cleaner and safer environment for agriculture.
\end{abstract}

Keyword: AUDPC; Defense enzymes; Environmentally friendly; Magnaporthe oryzae; Oryza sativa; Phenol 